\title{
La sociedad paralela: asistentes y asistidos
}

\author{
Danièlle Provansal \\ Universitat de Barcelona. Facultat de Geografia i Historia \\ Baldiri i Reixach, s/n. 08034 Barcelona. Spain
}

\section{Resumen}

La autora propone un análists de los mecanismos identitarios de afiliación y diferenciación utilizados entre los asistentes y asistidos sociales del mundo de la inmigración en la Cataluña de los últimos treinta años y que ha configurado lo que viene a denominar la "sociedad paralela». Con este objeto hará referencia a la génesis histórica del fenómeno y a una serie de vartables de tipo económico, cultural y político subyacentes que constituyen los motores reales de su evolución. Igualmente, considera necesario examinar cómo se efecría la producción social del extranjero, así como su categorización en este mismo escenario, revisando, a su vez, la pertinencia teórica de algunos conceptos aplicados en el marco de los diferentes modelos de integración de los inmigrantes interiores $y$ exteriores.

En el proceso de jerarquización simbólica y política inherente a toda dinámica de las representaciones de identidad y alteridad, se construyen las bases sociales del dominio que la sociedad de «acogidan ejerce sobre los grupos foráneos, interiorizando éstos el discurso dominante orientado a confundir diversidad cultural con diferencia esencial y legitimadora de las desigualdades de que son víctimas. La conciencia alienada del "Nosotros" en relación a los "Otros", que se traduce en determinadas arribuciones sociales de los grupos, se desarrolla en el cuadro de unas fronteras simbólicas cuyos límites sólo se moverán en función de los intereses objetivos que la mayoria nacional autóctona tenga de aumentar o disminuir la distancia social respecto a las minorías inmigradas. Por último, profundizando en la naturaleza de los intercambios entre ambos grupos (distinguiendo la especificidad de los inmigrantes extracomunitarios en el contexto señalado) para excraer las oportunas conclusiones, hace un breve repaso de lo que ha significado el proyecto político y la identidad cultural del nacionalismo catalán desde su aparición hasta nuestros días.

Palabras clave: sociedad paralela, nosotros-ellos, identidad cultural, minorias étnicas.

Abstract. The parallel society: social services givers and users

The author analyses the emergence of a parallel society among immigrants who arrived at Catalonia (Spain) on behalf of the recruitmenc of social services staff and users. The article shows the symbolic and political hierarchies established and the relationship between "Us" and the "Others". This has consequences in the confronting interests of the autochtonous population, nationalist in great proportion, and foreign immigrants, who 
are a social and cultural minority. The author finishes her writing by showing the kind of exchanges between both social gropus as regards the political project of Catalan nationalism. (Redacción)

Key words: parallel society, we-them, national identity, ethnic minorities.

\section{Sumario}

La dinámica de la identidad y de la alteridad

Proyecto político e identidad cultural

Los inmigrantes interiores y la adquisición de la catalanidad
Inmigración extra-comunitaria, sociedad paralela e invisibilidad social

Ilegalidad y etnicización

Bibliografía

Si se parte de la hipótesis según la cual todo proceso de construcción social del Nosotros y de los Otros tiende esencialmente hacia una naturalización de las definiciones y de las atribuciones identitarias — las definiciones relacionadas con el Nosotros y las atribuciones referidas a los Otros-, el análisis que se efectuará deberá remontarse a los orígenes del fenómeno o, dicho de otro modo, a las circunstancias históricas en cuyo desarrollo se ha incluido este juego de inclusión y de exclusión que esconde una relación real de naturaleza económica, social y política. Por consiguiente se deberá seguir las variaciones para comprender cómo y por qué esta relación objetiva se ha modificado y se ha traducido en un cambio en el sistema de autodefinición y de atribución.

La finalidad de este artículo es hacer resaltar, con ayuda de una ilustración concreta, cómo se instaura la dinámica de la identidad y de la alteridad. El ejemplo elegido es el de Cataluña a lo largo de las últimas tres décadas, las cuales constituyen un período que podríamos calificar en cierto sentido de paradigmático, puesto que está marcado por profundos cambios sociales y políticos, como la agudización de la reivindicación nacionalista, la transición a la democracia y la substitución de una inmigración interior por una inmigración procedente del Tercer Mundo.

Después de apuntar algunas propuestas generales de orden teórico y situar algunos hitos históricos destinados a referenciar el proceso de recuperación del hecho nacional catalán, así como a aclarar la dinámica identitaria a partir del sistema de representación que la funda, se intentará examinar globalmente la forma en que la sociedad catalana ha producido diferentes categorías de extranjeros en el curso de los últimos treinta años. Esta producción social será examinada a la vez desde un punto de vista objetivo, es decir, en relación a los condicionantes de la situación económica y política de Cataluña en el seno del Estado español, pero también en relación a la representación que ella tenía de sí misma en este conjunto, y analizar a través de este prisma los diferentes modelos de "integración». Hay que precisar que esta inmigración comprende dos períodos bien diferenciados que los estudios sobre el particular se com- 
placen en subrayar: el primero, entre los años 60 y 75, aproximadamente, que corresponde a la inmigración denominada interior, procedente de otras regiones de España, particularmente de Andalucía y Galicia, y la inmigración llamada exterior, de origen no europeo, que según las publicaciones aparecidas sobre el tema, le sucedería ${ }^{1}$.

En lugar de abordar estos dos tipos de migración, simplemente como dos etapas escalonadas en el tiempo, se va a tratar de analizarlas englobándolas en un sistema de representación en que la identidad y la alteridad no dejan de definirse recíprocamente y de manera diferente. Se trata, en definitiva, de ver cómo la distancia cultural, en principio medida en términos de grado, de lagunas a llenar, se convierte a continuación en una diferencia de "cualidad", es decir, insuperable. La polisemia del término integración nos permitirá utilizarlo de algún modo como analizador para marcar la diferencia entre los dos períodos, en particular y para ver, en cada caso, cuáles son las medidas que se desprenden y cuál es el modelo que las sostiene ${ }^{2}$.

\section{La dinámica de la identidad y de la alteridad}

Si se admite que la construcción de la identidad y de la alteridad no constituyen dos mecanismos independientes el uno del otro sino que son las dos caras de un mismo fenómeno que se crea alrededor de un mismo punto de vista o referente, el proceso que resultará, su doble de algún modo, se erigirá en torno a otro punto de vista o de un referente diferente, pero que quedará no obstante subordinado al primero, por lo menos dentro de la lógica de las relaciones que ello implica. Cuando esta interacción significa un intercambio de significados culturales y una reelaboración parcial de la representación del Nosotros y de los Otros, se inserta en un ámbito socialmente mediatizado en el que los sujetos implicados en dicha interacción se sitúan jerárquicamente en relación al "otro» tanto a nivel político como simbólico (caso de Cataluña y del Estado español).

Además, dentro de este proceso de jerarquización la imagen que un grupo tiene del otro interfiere en la autoimagen. Se crean así las condiciones para que el grupo formado por los «otros» interiorice las fronteras simbólicas que el grupo del "nosotros» ha construido al conferir a la diversidad cultural una función social de diferenciación. De este modo, todas las consecuencias que deri-

1. De hecho, según los datos recogidos en ciertas organizaciones no gubernamentales, esta distinción no es tan neta, puesto que es a lo largo de los años 65-70 que van llegando progresivamente, al menos, los primeros gambianos y los primeros marroquíes para sustituir a los trabajadores andaluces, principalmente en la agricultura especializada, en el sector de la construcción y en el de la hostelería.

2. Esto se realizó primeramente en el marco de una investigación de carácter europeo (Acción Integrada, 1991) coordinada por $\mathrm{G}$. Althabe por el lado francés y por C. Solé por la parte española, que se continúa actualmente en el seno de un grupo de trabajo constituido en torno al Institut Català d'Antropologia (Autorreproducción social, producción del extranjero). 
van de esta diferenciación no solamente estarán interiorizadas por el grupo que las soporta, sino que estarán vistas como parte de su responsabilidado, mejor dicho, como el efecto negativo de la diversidad cultural que ostenta. Esro corresponde de hecho a una esencialización de la diferencia equivalente a la esencialización de la identidad dificil de superar a pesar de las autodesignaciones del grupo de los "otros" que tienden a crear una cierta equivalencia en las representaciones y que, sobre todo, contribuyen a organizar un espacio estratégico en el campo de las mismas. Es lo que ocurre en el caso de la colonización: el grupo que en determinadas circunstancias se encuentra objetivamente capacitado para anular una frontera puede hacer asi emerger un conjunto arbitrario, reivindicando una unidad de destino y de cultura artificiales que impone al otro en función de sus propias necesidades sociales y políticas produciendo, al negar la identidad del otro, una alteridad alienada.

Por otra parte, a cada época corresponde una delimitación del Nosotros y de los Otros en la cual están implicadas relaciones reales y objetivas que se traduce a nivel simbólico en una representación espacial y/o temporal de la diferencia cultural más o menos grande en función de la necesidad que el grupo protagonista de esta delimitación tenga de acentuar o reducir la distancia social.

Finalmente, este juego de espejos entre identidad y alteridad no refleja forzosamente, tanto de un lado como del otro, la realidad total de estas relaciones, sino que implica más bien la doble representación que se ha hecho y su posible instrumentación en condiciones en adelante nuevas. Esta representación, por muy deformada o parcial que pueda parecer, sigue sin embargo la dinámica de la relación misma y, acorde con las circunstancias, reasigna el contenido que atribuye a la historia a partir de nuevas revalorizaciones o nuevos estereotipos que, a nivel imaginario, constituyen la transposición ética de exigencias concretas.

\section{Proyecto político e identidad cultural}

Este artículo no tiene por objetivo describir de manera detallada la historia de la recuperación del hecho nacional catalán. Sin embargo parece útil recordar algunos rasgos que contribuyen a subrayar su relativa particularidad ${ }^{3}$ y permiten dar, en este caso preciso, algún contenido al concepto de identidad.

Para empezar, conviene recordar que el movimiento de recuperación lingüístico y literatio de la "Renaixença», en el siglo XIX, no sirvió sólo para canalizar las primeras manifestaciones catatanistas, sino que ha dejado su huella en las corrientes nacionalistas que le siguieron. En efecto, a pesar de los matices ideológicos que los separan, todos, sin excepción, construiran su legitimidad a partir de la afirmación de la especificidad cultural y de la reivindicación de su

3. El término relativo sirve aque menos para matizar la noción de particularidad, sinónimo de historicidad, que de hacer resaltar ciersas constanres propias de todo fenómeno nacionalitario que, más allá de variantes regionales y contingentes, lo definen como robjeto sociológicon (C. Sole, 1976). 
plena y libre expresión. El catalanismo se ha construido, pues, como cultura de resistencia frente a un estado monolítico y opresor, buscando jugar el papel de catalizador de los diferentes sectores sociales que componen la sociedad catalana. Mientras que el factor étnico ha representado, a principios de siglo, una amenaza para este unitarismo cultural (en particular, con el movimiento lerrouxista), no ha significado to mismo durante el período de la fuerte inmigración interior de los años sesenta y setenta. La resistencia contra el franquismo se presentó a la vez como un combate por la democracia y como una reivindicación nacionalista en que la cultura ha constituido el elemento clave que lo ha conducidó.

Para explicar tal impacto tenemos que partir de un hecho objetivo sobradamente conocido: el potencial económico de Cataluña, superior al del resto del Estado pero, paradójicamente, sin venir acompañado del protagonismo político correspondiente. Esta contradicción, que se agravó durante las épocas de dictadura, no ha desaparecido nunca completamente en los otros períodos ${ }^{5}$. Esta superioridad económica habría representado en el siglo XIX, según los exégetas del nacionalismo, el elemento desencadenante de esta representación del Nosotros, que se afirma como reconstrucción histórica de una identidad propia ${ }^{6}$. A este respecto se puede citar una frase de Prat de la Riba:

Cuando un pueblo es rico, cuando no está completamente absorbido por la lucha por el pan cotidiano, siente las exigencias del espiritu y se entrega a ello. Cataluña, enriquecida por la industria, percibió que tenía un alma y entonces busco en los pergaminos del pasado sus aspiraciones para un mundo mejor.

La lengua es, sin embargo, el elemento esencial o el núcleo a partir del cual "el alma colectiva catalana" se define. A pesar de este esencialismo, el catalanismo representa un marco de pertenencia abierto al exterior, es decir, accesible a todas las personas que asumen los criterios de definición y los actualizan por su práctica. En esto se distingue del nacionalismo vasco, que se define por la ascendencia o, dicho de otra manera, por un origen consanguíneo común en que los patronímicos de los cuatro ascendientes aportan la prueba. En el

4. Bajo el franquismo una fracción de la alta burguesła catalana, en sintonía con el régimen, no participó de este unitarismo cultural, pero supo asumitlo posteriormente de manera oportunista y formal.

5. Cabe recordar que, hoy en día, el Estatuto de Autonomía político es valorado como insuficiente, primero con respecto a las perspectivas etropeas $y$, en segundo lugar, frente a las obligaciones fiscales que el Gobierno central impone a Cataluña, las cuales son percibidas como una transferencia abusiva de riqueza que alimenta el despilfarro y la impericia seculares del Estado español.

6. Las reivindicaciones identitarias tendrán, sin embargo, cierta dificultad para cuajar en un solo proyecto político, salvo en ciextos períodos de ctisis en que un bloque nacional se forma aunque de manera efinera. En general, el unitarismo cultural no ha venido acompañado de un unitarismo político. Los diferentes sectores sociales se han enfrentado a través del Estado, que algunos han utilizado en el sentido que conviene a sus propios intereses. 
caso catalán, la pertenencia no está sometida a ninguna limitación y se hace por un proceso de adquisición de los criterios de delimiración, mientras que en el segundo, se hace por adscripción, es decir, cerrado a los que no aportan la prueba de su filiación y que permanecerán como vascos de adopción. En resumen, la definición de la identidad catalana está adaptada a su historia, en la medida en que, como dice Pierre Vilar: «el mestizaje es la característica del pueblo catalán'. Acto seguido citamos una frase aparecida en la prensa y que expresa bien la síntesis entre estos dos principios de pertenencia identitaria aparentemente contradictorios:

El Mediterráneo constituye un viejo crisol étnico, todos tenemos algo de fenicio, de griego, de romano, de judío, de beteber, de íbero y de godo, pero una cosa es la estadística y otra distinta es el hecho de una pequeña sociedad que no solamente quiere vivir sino sobrevivir en sus esencias ${ }^{7}$.

De alguna manera, y según estas definiciones, el catalanismo, en tanto que pertenencia a un universo objetivamente particular, se fundaría sobre una esencia que podría conseguirse - a pesar de la contradicción conceptual que ello supone- a través de la lengua que constituiría el vector de adquisición esencial.

Efectivamente, lo dicho permite abordar el punto central de este artículo: el análisis de la inmigración en relación con el nacionalismo. Desde la edad media hasta el siglo XIX, Cataluña ha sido siempre un polo de atracción importante para los habitantes de otras regiones de Europa - particularmente la vecina Occitania- que huían del hambre, las guerras y la persecución religiosa, y posteriormente, como se sabe, por la gente procedente del sur y del oeste de España, en la época de la industrialización.

\section{Los inmigrantes interiores y la adquisicón de la catalanidad}

En la perspectiva de una revitalización del catalanismo, al final del franquismo y al principio de la democracia, esta inmigración no se ha considerado un obstáculo para el hecho catalán, en tanto que asumiera los criterios de catalanidad, particularmente la lengua, puesto que ella confirma su dinamismo y la capacidad asimiladora. En este sentido, el antiguo rector de la Universidad de Barcelona, Badia i Margarit, escribió en 1966: "la posibilidad de que los inmigrantes instalados en nuestra casa escapen a la asimilación es simplemente nula»?. Jaume

7. La Vanguardia, 7 de noviembre de 1989.

8. Esca afirmación puede ser matizada, en la medida en que las grandes familias catalanas se consticuyen sobre numerosas genetaciones de verdaderos linajes de renombre, subrayando así la función sacial del nacimiento e indicando sutilmente la diferencia no mentada pero real entre "viejos catalanes" y "nuevos catalanes".

Sin embargo, la prueba de la genealogía de los «cuatro ladosno, en otras palabras, de los cuatro abuelos, importante en todo vasco para afirmarse vasco, no tiene a primera vista el mismo rol en Cataluña.

9. Badia i Margarit, A. (1969). La llengua dels barcelonins. Barcelona: Edicions 62 . 
Russinyol atribuye esta fuerza asimiladora a las expectativas económicas que Cataluña ofrece a personas procedentes de regiones desheredadas: "el catalán es por esencia el instrumento de la promoción social y humanan, o bien ula asimilación lingüística significa la desproletarizaciónn (1976).

Se establece así un lazo entre la catalanización y el ascenso social, una engendrando al otro, lo cual puede ayudar a explicat en parte por qué la amalgama conceptual entre el nacionalismo generador per se de la democracia y la democracia definida en torno al hecho nacional persistirá a lo largo de los años venideros y fue recuperado por algunos sectores polfticamente dominantes que pusieron el acento sobre los aspectos lingüísticos de la integración.

El modelo de integración enfocado de esta manera se funda en una concepción de la diferencia cultural a partir de la representación del Nosotros y de la posición de inferioridad política de Cataluña en el seno del Estado español. Está visto sobre todo como una distancia a recorrer por el Otro para corregir el artificio creado por la imposición oficial del castellano y reparar así una injusticia histórica.

Algunos estudios sociologicos y de psicología social que se han hecho en esta época sobre la inmigración interior refiejan de cerca o de lejos esta inquietud y tienen por objeto enfocar la distancia cultural en sus manifestaciones cotidianas (C. Esteva Fabregat, 1984). Esta es examinada sobre todo desde la perspectiva lingǘstica que el tiempo, en el curso de una o dos generaciones, debe reducir y finalmente hacet desaparecer a través de medidas educativas y administrativas aptopiadas. Se puede considerar que este modelo de integración es esencialmente asimilador y se funda sobre una concepción de la diferencia vinculada a unas condiciones políticas e históricas particulares (Quadems d'Alliberament, 1978: Papers, número especial, 1980).

Por el lado de los antropólogos, los estudios sobre la identidad y la etnicidad estuvieron en auge a lo largo de los años ochenta. Al partir de un reconocimiento de la diferencia y sentar el principio de la diversidad cultural de los pueblos de España --casi siempre a partir de una perspectiva esencialista, más raramente a partir de un punto de vista constructivista y de la producción histórica del Nosotros y de los Otros en el seno de la dinámica social- son a menudo mediatizados por el contexto de las reivindicaciones autonomistas ${ }^{10}$; éstas no serán en lo sucesivo el patrimonio exclusivo de Cataluña y del País Vasco, sino que se extenderán al conjunto de España. Frente a estas manifestaciones particularistas, el estudio de las especificidades étnico-culturales se revela oportuna ${ }^{11}$. Sin embargo, se puede considerar que la mayor parte de los

10. Sobre el particular ver Prat (Coor), 1985-1986; Roque (Coor), 1988; Prat y orros,1991.

11. Mientras que en Cataluña y en el País Vasco el discurso etricista que reproduce el discurso nacionalista se apoya sobre la tradición culturalista y tiende a folklorizar la realidad social, en Andalucia y, en menor medida, en Galicia, este discurso se legirima a través del punto de vista tercermundista: las relaciones entre las regiones pobres y las regiones ricas españolas reproducen en miniatura las que, a escala mundial, son comúnmente denominadas relaciones notre-sur; en esre sentido es cuando la reivindicación identitaria se convierte en el instrumento revelador de la dependencia. 
estudios realizados en Cataluña no elaboran, o sólo un poco, un análisis crítico de los modelos de integración concebidos por los poderes públicos y se limitan en general a asumir sus implicaciones.

\section{Inmigración extracomunitaria, sociedad paralela e invisibilidad social}

En la época de la inmigración no comunitaria, este doble proceso de construcción del Nosotros y de los Otros se presenta de manera diferenciada. Por lo que respecta a la construcción del Nosotros, ciertos elementos vienen a modificar las condiciones. En primer lugar, el funcionamiento del Estatuto de Autonomfa, durante un cierto número de años, permite la afirmación de una sensibilidad propia para abordar la inmigración extracomunitaria. En segundo Iugar, la entrada de España en la Comunidad Europea y la construcción de una Europa de las regiones abre unas perspectivas totalmente nutevas. Un eje mediterráneo noroccidental se dibuja y en el cual Cataluña es uno de sus polos más importantes.

Por lo que concierne al proceso de construcción de los otros, hay que distinguir entre dos períodos: el que precede a la Ley de Derechos y Garantías de los Extranjeros de 1986 y el posterior a la misma.

Durante el primer período, existía una cierta circularidad entre permiso de trabajo y permiso de residencia, lo que permitía a la Administración practicar una política discriminatoria y oportunista, en función de la coyuntura. Por otro lado, durante el tiempo que duraban las diligencias era posible trabajar. Es así como los súbditos de paises vecinos - como los marroqú́estenían la posibilidad de ir y venir sin demasiadas dificultades, a pesar de o a causa de la aparente ineficacia administrativa.

En un estudio precedente (D. Provansal, 1991) se designó a este período como el de la invisibilidad social. En efecto, la sociedad catalana no ha tomado aún conciencia del cambio que se ha operado en ciertas ramas de actividad y de la substitución progresiva de los inmigrantes interiores por aquellos que provienen de otros continentes. Únicamente algunas asociaciones de beneficiencia guardan en sus archivos referencias detalladas de este primer período. Intentaremos, por tanto, tratar de dar algunas precisiones a partir del rol de mediadores que juegan estas organizaciones al lado de los ciudadanos del Tercer Mundo que se dirigen a sus servicios, dada la carencia de organismos públicos que se hagan cargo del tema en este momento.

Estas primeras demandas de asistencia tienen generalmente un carácter puntual. Emanan de individuos que en principio no tienen la intención de establecerse durante mucho tiempo en Cataluña, sino que buscan solamente los medios financieros para poder proseguir su viaje hacia otro país de Europa. Esta ayuda se concede, pues, a título humanitario a individuos considerados como personas necesitadas.

Algunos de estos inmigrantes de paso permanecen en el lugar sólo el tiempo necesario para ganar algún dinero. Efectivamente, las diferencias climáticas y el escalonamiento de los calendarios agrícolas entre las diversas regiones 
europeas favorece la aparición de estos trabajadores nómadas, que van y vienen a nivel continental en función de los cultivos y de las diferentes campañas de temporada. Esta movilidad que, según los defensores del modelo push and pull, adaptaria una fuerza de trabajo disponible a un mercado de trabajo europeo caracterizado por fuertes diferencias estacionales, se bloquea a partir de 1974, cuando la CEE empieza a aplicar una política de restricción para los ciudadanos procedentes de terceros países. A partir de esta fecha, estos inmigrantes de paso permanecen en el lugar donde habitan y, en un número cada vez mayor, acaban como empleados en el sector informal. Lo cual obliga enronces a los servicios de beneficiencia a una reconversión o, más bien, a una ampliación de sus funciones (trámites relativos a la regularización de los permisos de trabajo y de residencia, intermediarios cerca de los empleadores, etc.). Tratan de alguna manera de asegurar una inserción mínima, aunque precaria, puesto que la mayoría de los solicitantes de ayuda a las ONG no se han regularizado, ni tienen ninguna existencia legal frente a la Administración, lo cual les impide dirigirse a los servicios oficiales competentes. Cuando, a pesar de todo, lo hacen, son automáticamente encaminados hacia estas entidades de beneficiencia, conociendo perfectamente los poderes públicos el papel que ellas ocupan a este respecto. (Actualmente, el municipio de Barcelona da una subvención a algunas de estas organizaciones con el fin de que apliquen los programas de asistencia y de inserción decidida a nivel municipal.)

Es asf como se constituye progresivamente una sociedad paralela, dotada de sus propios instrumentos de funcionamiento pero, al mismo tiempo, abierta a la sociedad oficial, aquella que caracteriza el ideal del welfare state, mediante un mecanismo que podríamos calificar de retroactivo o, si se prefiere, de un doble proceso de reproducción de la marginalización y de su gestión, siendo ambos solidarios.

\section{Ilegalidad y etnicizacion}

A principios de los años ochenta algunas asociaciones dotadas de un servicio jurídico, al ser desbordadas por casos inextricables que no estaban preparadas para resolver, deciden denunciar públicamente la incoherencia administrativa. Esta campaña se suma a las exigencias de la CEE que presiona sobre España para que controle mejor las fronteras del sur de Europa.

Con la llamada Ley de Extranjería se establece un círculo vicioso entre el aumento de las restricciones y el aumento de los inmigrados en situación irregular. Los medios de comunicación, que hasta ese momento no se habían hecho eco de la cuestión, alertan a la opinión pública poniendo el acento sobre las entradas "ilegales", los residentes "clandestinos", así como algunos delitos ligados al pequeño tráfico de droga cometido por estos inmigrantes exteriores, marcando el fin del período de la invisibilidad social que, a partir de ahora, dejará de ser viable conceprualmente hablando.

Por otro lado, este nuevo marco legal significa una nueva categorización de los extranjeros que no tienen sus papeles en regla. Antes de la aparición de 
ta Ley, los ciudadanos extracomunitarios que no tenían un contrato de trabajo previo a su llegada a España no podían, en principio, trabajar. Sin embargo, en la práctica, podían trabajar temporalmente antes de conseguir los permisos de trabajo y de residencia correspondientes con la simple presentación de una carta con promesa de contratación. En realidad, estos permisos, cuando se conseguían, llegaban a expirar poco tiempo después de su concesión, dado que se establecía un círculo vicioso entre ambos, no pudiendo obtener el primero sin tener el segundo y viceversa.

Estos inmigrantes trabajaban en condiciones precarias pero, salvo medidas discrecionales de los poderes públicos, poco usuales, podían permanecer aquí. A partir de 1986 esto viene a resultar imposible para los inmigrantes que no entran en ninguna de las categorías con derecho a permiso previstas por la ley. Esto trae como consecuencia su inmersión inmediata en la economía sumergida. Asi, en lugar de mejorar la situación del conjunto de los extranjeros, esta ley crea categorías con derechos diferenciados, de modo que, en función del continente de origen y la nacionalidad, define restricciones más o menos severas.

Desde el punto de vista del proyecto de consolidación nacional y del modeto de integración que ello implica, este fenómeno de visibilidad obliga a una redefinición de los términos o, dicho de otra manera, a la adopción de un modelo de inserción económica controlada, de "puerta estrecha", podríamos decir, que se acompaña de un modelo de inserción social equivalente y designado siempre bajo el vocablo de integración, pero que se limita a optimizar los recursos disponibies en materia de asistencia. Es la razón por la que, según esta lógica, las organizaciones no gubernamentales no dejan de ampliar sus funciones. La asistencia familiar, los cursos de alfabetización, así como las actividades vinculadas a las cuituras de origen, los ponen de lleno en contacto con una realidad que sobrepasa el marco estricto del trabajo y que, a través de la organización de la vida doméstica y privada, hace emerger de repente un «más allán cultural.

En este estado de cosas, la racionalización de lo que deja de estar marcado por el signo de la beneficiencia y se convierte en acción social, aunque de carácter privado, pasa por una etnicización de las diferentes categorías de asistidos. Según las personas entrevistadas, esta diferenciación a partir del origen étnico, sería debida a una preocupación por la operatividad, con el fin de conocer mejor la especificidad de las culturas de origen y de adaptar, en consecuencia, las diferentes modalidades de la asistencia, pero uno se puede preguntar si no correspondería al mismo tiempo a un modelo de integración diferencial, en función de la distancia histórico-cultural, tal como es percibida socialmente. Al tratar con individuos que colocan en un subgrupo que, de ahora en adelante, van a representarles y que está connotado étnicamente, estas diferentes asociaciones de ayuda contribuyen, a pesar de ellas, a la formación de fronteras étnico-cuiturales. Por ejemplo, cuando ellas buscan favorecer las manifestaciones culturales propias de los países de origen de los inmigrantes, lo hacen alegando la eventualidad del retorno, particularmente de la segunda genera- 
ción. Ahora bien, el desconocimiento que la mayor parte de asistentes sociales y de los educadores tienen de estas culturas, al menos en un primer momento, es reemplazado progresivamente por un conocimiento superficial, a veces instrumental, a veces folklorizado, en cualquier caso, raramente vivido.

Por otro lado, los poderes públicos (principalmente los servicios sociales que dependen de la Generalitat y del Ayuntamiento de Barcelona) han desarroliado recientemente toda una serie de programas de intervención para los grupos marginalizados, en los cuales las minorías étnicas figuran al lado de toxicómanos, de la tercera edad, de la juventud y de los sectores sociales considerados como población "de riesgo" (drogadictos, delincuentes, etc.) (c.f. M. Muñoz, 1991).

En resumen, las asociaciones de ayuda juegan un papel de mediador activo entre la sociedad global y los inmigrantes extracomunitarios, en la medida en que ellas contribuyen a construirlos como categoría social y a resituarlos en un orden étnico-cultural a través del cual se canaliza su visibilidad social. El desarrollo de este proceso se acompaña, a nivel de la sociedad global, de la emergencia de una nueva concepción de la diferencia: ella corresponde menos a los presupuestos evolucionistas de la época de la inmigración interior, los cuales se expresaban mediante el reconocimiento de una distancia cultural más o menos grande, pero siempre reductible, y se funda más sobre una perspectiva cualitativa, o si se prefiere substantiva, que viene a reforzar los prejuicios y los estereotipos implícitos del sistema de representación colectivo. En suma, el Otro continúa siendo "otron, sea cual sea su capacidad de integración en la sociedad receptora y en su cultura.

Es así como el reconocimiento de la alteridad, bajo la forma de minorías étnicas o de comunidades de extranjeros procedentes deł Tercer Mundo, se convierte en un componente esencial de la nueva estructuración de la sociedad catalana. La idea de una sociedad marcada por el signo de la pluralidad y de la necesidad de distribuir espacios multiculturales está a punto de emprender su camino. Prueba de ello es la insistencia puesta en la necesidad de un proyecto social plural por parte de las instancias oficiales.

¿La ampliación de la base demográfica de Cataluña, necesaria para su nuevo rol de polo macro-regional a nivel europeo, no implica una extensión de su base pluriétrica a través de una nueva forma de integración controlada y ecléctica de las diferentes comunidades de inmigrantes?

\section{Bibliografía}

ALTHABE, G. (1991). "La construction sociale de l'étranger stigmatisem. Comunicación presentada en el seno del seminario sobre La inmigración no comunitaria en el sur de Europa. Barcelona (dactilografiado).

BALCELLS, A. (1977). Cataluña Contemporánea I (Siglo XIX) y II (1900-1939), Siglo XXI.

ESTEVA FABREGaT, C. (1982). Estado, etnicidad y biculturalismo. Barcelona: Península. MUNo\%, M. (1991). De la beneficiencia al Estado del bienestar: el caso de los negroafricanos en Cataluña (no publicado). 
PEATTIE, L. (1987). "An idea in good currency and how it grew: the informal sectorn. World Development, 7.

PRADA M. DE (1990). "España de país de emigración a país de inmigración". En Movimientos Humanos en el Mediterráneo Occidental. Generalitat de Catalunya. Institut Català d'Estudis Mediterranis, p. 209-242.

PRAT, J. (1956-1986). "Trenta anys de Literatura Antropologica espanyola". Arxiu d'Etnografia de Catalunya, núm. 4-5.

PRATS, L1. (1988). El mite de la tradició popular, els origens de l'interes per la cultura tradicional a la Catalunya del segle XX. Barcelona: Edicions 62.

PROVANSAL, D. (1991). "Nazionalismo e relazioni intererniche: l'esemplo delle comunità africane in Catalogna". En CHIOzZI, P. (ed.). Antropologia urbana e relazio$n i$ interetniche. Florencia: Angelo Pontecorboli ed.

PUJADAS, J.J. y CuCO, J. (Coors.). (1990). Identidades colectivas, etnicidad y sociabilidad en la Peninsula Iberica. Generalirat Valenciana.

PUjOL, J. (1976). La immigracib: problema i esperança de Catalunya. Barcelona: Nova Terra.

QUADERNS D'ALIBberament (1978). La inmigració als Paísos Catalans, núm. 2-3. Barcelona: Edicions de la Magrana.

RFCiO, A. (1986). "Economía sumergida y transformación de las relaciones laborales en Españas. Papers, núm. 27, p. 131-157.

ROQUE, M.A. (Coord.), (1988). Encontre d'Antropologia i diversitat hispànica Barcelona: Generalitat de Catalunya.

- (1990). Moviments Humans en el Mediterrani Occidental. Barcelona: Generalitat de Catalunya.

RUSINYOL, J. (1974). Le probleme national catalan. Mouton.

SAYAD, A. (1991). L'immigration ou les paradoxes de l'alterite. Ed. Universitaires.

SOLE, C. (1981). La integración sociocultural de los inmigrantes en Cataluña. Madrid: Centro de Investigaciones Sociológicas.

SOLE, C. y HERRERA, E. (1991). Trabajadores extranjeros en Cataluña. Madrid: Centro de Investigaciones Sociológicas.

STrubelL, M. (1984), "Evolució sociolingüística a Catalunya». Papers, núm. 24, p. $27-47$.

VILAR, P. (1962). La Catalogne dans /'Espagne modeme. Recherches sur les fondements économiques des structures nationales. París: EVEPEN. 